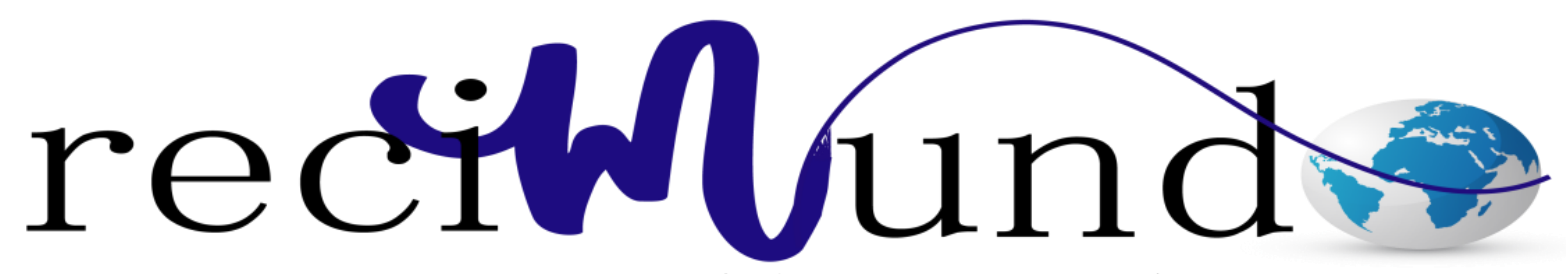

Revista Cientifica Mundo de la Investigación y el Conocimiento

\begin{abstract}
Jenny Irene Plasencia Criollo ${ }^{\text {a }}$; Fátima Victoria Gualotuña Cueva ${ }^{\text {b; }}$ María Jhaneth
\end{abstract}
Delgado Angamarca ${ }^{c}$; Sixto Duberli Paucar Llapapasca ${ }^{d}$

Manejo en intoxicación por metanol

Management in methanol poisoning

Revista Científica Mundo de la Investigación y el Conocimiento. Vol. 3 núm.3, septiembre, ISSN: 2588-073X, 2019, pp. 842-869

DOI: $10.26820 /$ recimundo/3.(3).septiembre.2019.842-869

URL: http://recimundo.com/index.php/es/article/view/551

Código UNESCO: 3205 Medicina Interna

Tipo de Investigación: Artículo de Revisión

Editorial Saberes del Conocimiento

Recibido: 15/05/2019

Aceptado: 23/06/2019

Publicado: 30/09/2019

Correspondencia: jenny.plasencia@hotmail.com
a. Médico Cirujano; Investigador Independiente; Guayaquil, Ecuador; jenny.plasencia@ @otmail.com
b. Médico; Investigador Independiente; Guayaquil, Ecuador; faty.cuev@gmail.com
c. Médico; Investigador Independiente; Guayaquil, Ecuador; majha-2@ hotmail.com
d. Especialista en Medicina Familiar y Comunitaria; Medico General; Investigador Independiente; Guayaquil, Ecuador; sidupall224@ hotmail.com 


\section{Manejo en intoxicación por metanol}

Vol. 3, núm. 3., (2019)

Jenny Irene Plasencia Criollo; Fátima Victoria Gualotuña Cueva; María Jhaneth Delgado

Angamarca; Sixto Duberli Paucar Llapapasca

\section{RESUMEN}

El metanol, también conocido como alcohol metílico, alcohol de madera; es el alcohol más sencillo. Es un líquido incoloro con un fuerte olor, extremadamente tóxico que se puede absorber a través de los ojos, la piel, los pulmones y el sistema digestivo. La sobreexposición puede causar la muerte. La ceguera es común y, a menudo, permanente, a pesar de los cuidados médicos. Se usa en muchas industrias. Este trabajo investigativo se enfoca, principalmente, en la revisión de la literatura cientificoacadémica disponible y, en tanto sea posible, de data reciente, respecto a la intoxicación (o envenenamiento) por metanol, en general, haciendo un mayor énfasis en cuál sería su manejo o tratamiento. En efecto, los resultados obtenidos sirvieron para exponer en concreto sobre el manejo (tratamiento) de este tipo de complicaciones, sin embargo, igualmente se pudo aportar otros aspectos relevantes que facilitan la comprensión del tema en cuestión. Las conclusiones derivaron, por una parte, en el reconocimiento de que esta enfermedad es relativamente rara pero que no deja de prestársele suma importancia; el posible carácter multisintomático a nivel clínico, asociado a la gravedad de la intoxicación y la particularidad de cada caso; y que el ácido fórmico (principal metabolito del metanol) frecuentemente es el primer responsable de la mayoría de las anormalidades clínicas de esta enfermedad. Finalmente se resalta la comprensión de que la idea sostenida en la especialidad médica es la de manejar esta condición con terapias combinadas, preferiblemente, en las que se deben incluir: la administración de un inhibidor, y a falta de este, el etanol por su efectividad. Así mismo, otros sostienen la premisa de restringir la hemodiálisis a circunstancias específicas y severas. En síntesis, todo lo anterior en definitiva favorecerá significativamente la pronta recuperación del paciente, la delimitación en cuanto a la exposición medicamentosa y la reducción de los días de hospitalización.

Palabras Claves: Tóxico; Sobreexposición; Multisintomático; Metabolito; Anormalidades. 


\title{
Manejo en intoxicación por metanol
}

Vol. 3, núm. 3., (2019)

Jenny Irene Plasencia Criollo; Fátima Victoria Gualotuña Cueva; María Jhaneth Delgado Angamarca; Sixto Duberli Paucar Llapapasca

\begin{abstract}
Methanol, also known as methyl alcohol, wood alcohol; It is the simplest alcohol. It is a colorless liquid with a strong, extremely toxic smell that can be absorbed through the eyes, skin, lungs and digestive system. Overexposure can cause death. Blindness is common and often permanent, despite medical care. It is used in many industries. This research work focuses mainly on the review of the available scientific and academic literature and, as far as possible, of recent data, regarding methanol poisoning (or poisoning), in general, with greater emphasis on its management or treatment In fact, the results obtained served to express in particular about the management (treatment) of this type of complications, however, other relevant aspects that facilitate the understanding of the subject in question could also be provided. The conclusions derived, on the one hand, in the recognition that this disease is relatively rare but does not cease to be of utmost importance; the possible multisymptomatic character at the clinical level, associated with the severity of the poisoning and the particularity of each case; and that formic acid (the main metabolite of methanol) is often the main cause of most of the clinical abnormalities of this disease. Finally, it is highlighted the understanding that the idea sustained in the medical specialty is to manage this condition with combined therapies, preferably, which should include: the administration of an inhibitor, and in the absence of this, ethanol for its effectiveness. Likewise, others support the premise of restricting hemodialysis to specific and severe circumstances. In summary, all of the above will definitely favor the rapid recovery of the patient, the delimitation in terms of drug exposure and the reduction of hospitalization days.
\end{abstract}

Key Words: Toxic; Overexposure; Multisymptomatic; Metabolite; Abnormalities. 


\section{Manejo en intoxicación por metanol}

Vol. 3, núm. 3., (2019)

Jenny Irene Plasencia Criollo; Fátima Victoria Gualotuña Cueva; María Jhaneth Delgado

Angamarca; Sixto Duberli Paucar Llapapasca

\section{Introducción.}

El Instituto Nacional de Seguridad y Salud Ocupacional de los Estados Unidos de América (NIOSH, por sus siglas en inglés) indica que el metanol (alcohol metílico = $\mathrm{CH} 3 \mathrm{OH}$ ) es un líquido incoloro con un fuerte olor. Es una sustancia venenosa que se puede absorber a través de los ojos, la piel, los pulmones y el sistema digestivo. La sobreexposición puede causar la muerte. Los trabajadores pueden verse perjudicados por la exposición al alcohol metílico. El nivel de daño depende de la dosis, la duración y el trabajo realizado. El alcohol metílico se usa en muchas industrias. Se utiliza en solventes, deshielo y en la fabricación de plásticos, poliésteres y otros productos químicos. Algunos ejemplos de trabajadores en riesgo de exponerse al alcohol metílico incluyen los siguientes: trabajadores de fábricas en plantas que fabrican plásticos, trabajadores del transporte expuestos al deshielo de vehículos, trabajadores que trabajan en industrias de combustibles alternativos, trabajadores expuestos a ciertos solventes o agentes de limpieza. (NIOSH, 2019)

El metanol es extremadamente tóxico. Tan solo 2 cucharadas $(30 \mathrm{ml})$ pueden ser mortales para un niño. Alrededor de 2 a 8 onzas $(60$ a $240 \mathrm{ml})$ pueden ser mortales para un adulto. La ceguera es común y, a menudo, permanente, a pesar de los cuidados médicos. La ingesta de metanol afecta varios órganos. El daño a estos órganos puede ser permanente. El pronóstico para la persona depende de la cantidad de tóxico ingerida y de la prontitud con que se reciba el tratamiento. (Borke, 2019)

En los seres humanos la toxicidad se produce a través de sus productos metabolitos, formaldehído y, especialmente, el ácido fórmico que llega a producir disfunción neurológica, 


\section{Manejo en intoxicación por metanol}

Vol. 3, núm. 3., (2019)

Jenny Irene Plasencia Criollo; Fátima Victoria Gualotuña Cueva; María Jhaneth Delgado Angamarca; Sixto Duberli Paucar Llapapasca

metabólica, respiratoria, renal y cardiovascular; en algunos casos se puede producir ceguera permanente y muerte. El metanol es absorbido y rápidamente distribuido en el organismo; no se une a proteínas y tiene un volumen de distribución de 0,6-1/kg de peso. La intoxicación por metanol no es exclusividad de los pacientes alcohólicos crónicos, que consumen alcohol de dudosa procedencia, sino que, además pueden presentarse en personas que manipulan productos, solventes o sustancias que contienen el alcohol metílico. Representa el 0,3\% de todas las que, a su vez, puede ser accidental o con fines autolíticos y el 1,5\% de las intoxicaciones por alcohol. A pesar del tratamiento inmediato, la mortalidad es alrededor de $20 \%$ y una proporción similar tiene daño visual residual, el retraso en la terapéutica específica incrementa la morbimortalidad (Contreras, Lira, Contreras, \& Gala, 2019)

Los casos de intoxicación aguda por metanol en el Ecuador principalmente ocurren por consumo de licor adulterado; en los últimos años se han registrado más de 300 personas intoxicadas entre el año 2011 y tercer trimestre del año 2012, que cobro la vida de más de 50 personas y muchas otras quedaron con secuelas principalmente visuales. (Ministerio de Salud Pública Ecuador, 2017)

En base a lo antes expuesto, con este trabajo investigativo se persigue hacer una revisión, principalmente, a la literatura cientificoacadémica que se encuentre disponible y, en tanto sea posible, reciente, a fines de exponer sobre intoxicaciones (o envenenamientos) por metanol, en general, haciendo un mayor énfasis en cuál sería su manejo o tratamiento.

\section{Materiales y métodos.}




\section{Manejo en intoxicación por metanol}

Vol. 3, núm. 3., (2019)

Jenny Irene Plasencia Criollo; Fátima Victoria Gualotuña Cueva; María Jhaneth Delgado

Angamarca; Sixto Duberli Paucar Llapapasca

Efectuar una revisión a la literatura cientificoacadémica reciente disponible mediante búsqueda de referencias bibliográficas en diversas bases de datos, entre las que figuran: NCBI, SciELO, MedlinePlus, BVS, Embase, PubMed, Dialnet, entre otras; usando las expresiones "intoxicación por metanol", "manejo de intoxicación por metanol" y "tratamiento de la intoxicación con metanol", ha sido uno de los objetivos del presente trabajo, para ello, básicamente se ha dispuesto de varios computadores personales con conexión a internet, como materiales principales de trabajo, y sumado a eso, algunos textos rescatados de la biblioteca universitaria y otras particulares.

Debido a que otro de los principales objetivos ha sido el de identificar y referir cuáles serían los preceptos recientes con acceso completo dentro del universo de las fuentes bibliográficas que indiquen cuál vendría siendo el tratamiento para esta complicación, principalmente se han considerado escoger: artículos científicos, guías clínicas, e-books, ensayos clínicos, protocolos, revisiones sistemáticas con o sin metaanálisis, boletines y/o folletos de instituciones privadas, formales u oficiales, tesis de posgrado y doctorado, noticias científicas y demás documentos e informaciones asumidos bajo propio criterio del equipo investigador como de interés y considerable valor de la evidencia científica, publicados preferiblemente en los últimos 7 años (incluyendo el presente), es decir, desde 2012 hasta el 2019, salvo algunas excepciones.

Se desestimaron estudios de cohorte, casos y controles, series y reportes de casos, editoriales, cartas al editor y otros tipos de materiales bibliográficos que, a juicio de este equipo, 


\section{Manejo en intoxicación por metanol}

Vol. 3, núm. 3., (2019)

Jenny Irene Plasencia Criollo; Fátima Victoria Gualotuña Cueva; María Jhaneth Delgado Angamarca; Sixto Duberli Paucar Llapapasca

se consideraron de escaso valor científico académico o nivel de evidencia percibido de en sus contenidos.

El resultado obtenido tras la búsqueda en dichas bases de datos, ascendió a más de un centenar de miles de referencias bibliográficas, en conjunto, lo que evidenció la existencia de suficiente contenido bibliográfico desarrollado en base a esta temática, sin embargo, fue necesaria la depuración de los mismos en función de los objetivos previamente planteados y a razones tales como: idioma español, relevancia, correlación temática y fecha de publicación en el lapso antes mencionado, fundamentalmente.

Paralelamente, se adelantó una búsqueda sin limitación cronológica ni de nivel de evidencia para identificar información igualmente relevante, por una parte, distinta a las recomendaciones para dicho manejo, pero por la otra, necesaria para el desarrollo de la presente temática y revisión, siendo a partir de allí que el equipo investigador le da la correspondiente lectura crítica y análisis interpretativo a toda la evidencia en definitiva seleccionada, resultando dicho proceso en el fundamento de las ideas aquí expresadas de manera consensuada.

La inclusión o exclusión de cada una de las referencias en la presente revisión se definió igualmente por el consenso todos los participantes en este proceso investigativo, y de la misma manera fueron resueltas las decisiones disímiles.

Resultados. 


\section{Manejo en intoxicación por metanol}

Vol. 3, núm. 3., (2019)

Jenny Irene Plasencia Criollo; Fátima Victoria Gualotuña Cueva; María Jhaneth Delgado

Angamarca; Sixto Duberli Paucar Llapapasca

El más simple de los alcoholes es el metanol. Esta sustancia tóxica puede ocasionar dolores de cabeza, mareos, náuseas, vómitos e inclusive la muerte, en un individuo que se exponga al mismo en concentraciones elevadas. Igualmente, puede ocasionar ceguera si la persona es sometida a una exposición aguda. De la misma manera, una exposición crónica puede ser causa de daños al hígado.

Son múltiples los usos que se le puede dar al metanol. Como disolvente industrial es utilizado como materia prima en la elaboración de formaldehido. El metanol también se emplea como anticongelante en vehículos, combustible de bombonas de camping-gas, disolvente de tintas, tintes, resinas y adhesivos. El metanol puede ser también añadido al etanol para hacer que éste no sea apto para el consumo. (GreenFacts, 2019)

Basándose únicamente en su sabor y olor, es imposible diferenciarlo del alcohol etílico. La intoxicación por metanol es posible de forma secundaria a la ingesta de bebidas alcohólicas de origen desconocido, es decir, que se ingiera metanol en lugar de etanol. El tracto digestivo lo absorbe rápidamente. En la sangre, puede llegar a su máxima concentración entre los 30 y 60 minutos posterior a su consumo. El proceso de metabolización de éste disolvente ocurrido en el hígado, es debido a la actividad de la alcohol deshidrogenasa, que por cierto, en comparación con el etanol, ocurre mediante un procesamiento más lento; en definitiva transformándose a formaldehído y ácido fórmico, siendo ambos metabolitos los causantes de acidosis metabólica grave y de daños orgánicos. La acumulación de ácido láctico se ve incrementada por la acidosis que se desarrolla, en razón de una hipoxia celular secundaria. Para que pueda hablarse de una 


\section{Manejo en intoxicación por metanol}

Vol. 3, núm. 3., (2019)

Jenny Irene Plasencia Criollo; Fátima Victoria Gualotuña Cueva; María Jhaneth Delgado Angamarca; Sixto Duberli Paucar Llapapasca

dosis fatal de metanol, esta debe tratarse de una concentración de entre 0,5 y $1 \mathrm{ml} / \mathrm{kg}$. (Empendium, 2019)

Según Copaja (2018) la estructura del metanol (alcohol) es $\mathrm{CH} 3 \mathrm{OH}$, que bien puede usarse como solvente o también en procesos de síntesis de nuevas moléculas. Existen una diversidad de productos en los que este alcohol puede hallarse como componente, tales como: adhesivos, pinturas, tintas, removedores de pintura, productos de limpieza, resinas, anticongelantes, productos fotográficos, entre otros. Resulta que vendría siendo precisamente su versatilidad industrial lo incremente el riesgo a su exposición, pues podría ser mediante la inhalación de sus vapores, el contacto con mucosas, o también, mediante la piel, al manipular inadecuadamente aquellos productos que contienen esta sustancia.

Este autor se atreve a confirmar que, es mayormente en la población adulta que la intoxicación aguda ocurre como causa principal, y es debido al consumo de licor adulterado, pues el metanol, al provocar efectos muy similares a los de embriaguez por etanol, es considerada una sustancia ideal para la adulteración de las bebidas alcohólicas.

Es un caso poco frecuente de intoxicación que resulta en acidosis metabólica con un incremento de la brecha aniónica debido a la producción de ácido fórmico (tóxico para el sistema nervioso central) mediada por la alcohol deshidrogenasa (ADH). Está caracterizada por mareos, nauseas, vómitos, confusión, acidosis metabólica, perturbaciones visuales (que si no se tratan pueden conducir a ceguera), coma y fallecimiento (debido a insuficiencia respiratoria). (Orphanet, 2019) 


\section{Manejo en intoxicación por metanol}

Vol. 3, núm. 3., (2019)

Jenny Irene Plasencia Criollo; Fátima Victoria Gualotuña Cueva; María Jhaneth Delgado

Angamarca; Sixto Duberli Paucar Llapapasca

Absorción y Distribución de metanol en el organismo

Retomando la obra de Copaja (2018) se comprende que el metanol también es absorbido velozmente por todas las vías, es decir, la oral, dérmica e inhalatoria. Por vía oral se absorbe completamente entre los 30 a 90 minutos de exposición, tiempo en el cual alcanza su máxima concentración plasmática. Seguidamente, el metanol perfunde menos lento en todos los órganos durante el proceso de distribución, sin unirse a las proteínas, razón por la cual se puede dializar. Este volumen de distribución es de 0,6 a 0,7 L/Kg, por ello, su penetración en tejidos es baja.

Entonces, es a nivel plasmático que circula la mayor parte del metanol, atravesando la barrera hematoencefálica y siendo metabolizado por el hígado lentamente. En promedio, su subsistencia fluctúa de entre las 12 y 24 horas.

Participación del metabolismo en la toxicidad del metanol y su eliminación

Es importante comprender que el metanol en el cuerpo humano puede ser excretado en un $10 \%$ aproximadamente sin generar cambios mediante la función renal y pulmonar. Esto quiere decir que "el $90 \%$ restante es metabolizado en el hígado mediante dos procesos enzimáticos". (Copaja, 2018) Éste detalla que en el primer proceso enzimático se genera la conversión del metanol a formaldehido mediante la enzima alcohol deshidrogenasa, mientras que luego, en el segundo proceso oxidativo, producido por la aldehído deshidrogenasa es que ocurre el paso de formaldehído a ácido fórmico que se elimina por vía urinaria.

En cuanto a los efectos de estos agentes, metanol y sus metabolitos, se describe que el metanol per se sólo produce depresión del sistema nervioso central (SNC), siendo sus 


\section{Manejo en intoxicación por metanol}

Vol. 3, núm. 3., (2019)

Jenny Irene Plasencia Criollo; Fátima Victoria Gualotuña Cueva; María Jhaneth Delgado Angamarca; Sixto Duberli Paucar Llapapasca

metabolitos los responsables del efecto tóxico. En este sentido, aunque algunos reportes indican que el formaldehido carece de efectos debido a su rápida biotransformación en ácido fórmico (J.

Roldan et. al., 2003), estudios posteriores atribuyen a este metabolito daño oxidativo con un impacto en el ciclo celular (M. Pohanka M., 2016). Por otro lado, el ácido fórmico además de inducir una acidosis metabólica, inhibe la citocromo oxidasa, interfiriendo así directamente con el transporte de electrones en la cadena respiratoria inhibiendo la función mitocondrial en la retina y aumentando el estrés oxidativo. En términos clínicos se reportan desórdenes visuales, ceguera, convulsiones, coma, y puede ocurrir la muerte. Los pacientes describen alteraciones visuales, como visión borrosa y colores alrededor de los objetos. En el examen del fondo de ojo se puede evidenciar hiperemia del disco óptico. Asociado a lo anterior, la midriasis precoz y no reactiva es un signo de mal pronóstico y significa pérdida irreparable de la función visual. Dado que el formaldehido y el ácido fórmico son los responsables del efecto tóxico del metanol, evitar su metabolismo constituye una estrategia clásica en cuadros de intoxicación. Tal como se muestra en la figura 1, el etanol compite por la Alcohol deshidrogenasa siendo la afinidad de este último 20 veces mayor, constituyendo una alternativa de tratamiento en casos de intoxicación. Por otro lado, el uso de folatos favorece la biotransformación del ácido fórmico a CO2 y agua, disminuyendo sus niveles de concentración y por ende su efecto dañino (McMartin K., et al., 2015). En términos de su toxicidad, La dosis tóxica del metanol es de 10-30 mL (100 mg/Kg), aunque ingestas menores han causado ceguera. Es letal por encima de 60-240 mL (340 mg/kg). (Copaja, 2018, p. 2-3)

\section{Síntomas}




\section{Manejo en intoxicación por metanol}

Vol. 3, núm. 3., (2019)

Jenny Irene Plasencia Criollo; Fátima Victoria Gualotuña Cueva; María Jhaneth Delgado

Angamarca; Sixto Duberli Paucar Llapapasca

Antes de que el metanol se metabolice, provoca depresión del SNC y síntomas de embriaguez de manera similar al etanol. Una vez desarrollada la intoxicación se observan: alteraciones de la conciencia, pudiendo llegar hasta el coma, hiperventilación (respiración de Kussmaul), reducción de la presión arterial, taquicardia, y en ocasiones bradicardia. En las intoxicaciones graves pueden aparecer convulsiones, insuficiencia respiratoria aguda, y a veces, pancreatitis aguda. Es un hallazgo específico de la intoxicación por metanol (sobre todo en los casos de diagnóstico tardío) la visión borrosa, la cual se debe al edema y/o a daños en la retina y en el nervio óptico (en la mayoría de los casos el daño ocular es permanente). (Empendium, 2019)

Por su parte Borke (2019) en detalle describe que los síntomas en: las vías respiratorias y pulmones; pueden ser: dificultad respiratoria, Paro respiratorio; Ojos: ceguera, completa o parcial, (a veces descrita como: ceguera de la nieve), visión borrosa, dilatación (ensanchamiento) de las pupilas; corazón y vasos sanguíneos: presión arterial baja; sistema nervioso: comportamiento agitado, coma (inconsciente), confusión, dificultad para caminar, mareo, dolor de cabeza, crisis epiléptica; piel y uñas: uñas y labios azulados; estómago e intestinos: dolor abdominal (fuerte), diarrea, problemas con la función hepática, incluso ictericia (piel amarilla) y sangrado, náuseas, pancreatitis (náuseas, vómito, y dolor abdominal) vómitos (a veces con sangre); otros: fatiga, calambres en las piernas, debilidad.

\section{Diagnóstico}

\section{Examen toxicológico}




\section{Manejo en intoxicación por metanol}

Vol. 3, núm. 3., (2019)

Jenny Irene Plasencia Criollo; Fátima Victoria Gualotuña Cueva; María Jhaneth Delgado Angamarca; Sixto Duberli Paucar Llapapasca

Concentración del metanol en el plasma o en el suero y en la orina (prueba necesaria para confirmar la intoxicación; si no es posible realizar la determinación de metanol de urgencia, hay que realizarla lo más rápidamente posible para monitorizar la eficacia de la terapia); una concentración en sangre $>20 \mathrm{mg} / \mathrm{dl}$ se considera tóxica; con niveles de $\sim 100 \mathrm{mg} / \mathrm{dl}$ habitualmente se produce daño en los nervios ópticos; una concentración >150 mg/dl es letal, aunque se pueden describir muertes con concentraciones menores, dependiendo de la susceptibilidad de los pacientes. En la fase con desarrollada acidosis profunda la concentración de metanol en la sangre puede no ser elevada (incluso puede ser indetectable) porque ya ha sido metabolizado. En este caso la gravedad de la intoxicación se evaluará sobre la base de la severidad de la acidosis y de la amplitud del anión gap y de la brecha osmótica.

Otros

gasometría arterial (a menudo $\mathrm{pH}<7,0, \mathrm{HCO} 3<10 \mathrm{mmol} / \mathrm{l}$ ), anión gap (aumento), brecha osmótica (aumento), concentración del ácido láctico, concentración de electrólitos en suero (Na, K), actividad de aminotransferasas, amilasa y CK en suero, examen de fondo de ojo (el daño de la imagen no es distintivo). (Empendium, 2019)

\section{Consideraciones Diagnósticas}

Las condiciones a considerar en el diagnóstico diferencial de la intoxicación por metanol incluyen las siguientes:

- Envenenamiento por arsénico

- Intoxicación por cocaína 
Manejo en intoxicación por metanol

Vol. 3, núm. 3., (2019)

Jenny Irene Plasencia Criollo; Fátima Victoria Gualotuña Cueva; María Jhaneth Delgado

Angamarca; Sixto Duberli Paucar Llapapasca

- Envenenamiento por inhalación

- Convulsiones parciales complejas

- Intoxicación por etilenglicol

- Envenenamiento por monóxido de carbono

- Seudoconvulsiones

- Cualquier causa de estado mental alterado con acidosis y posible colapso cardiovascular. (Korabathina, Benbadis, \& Likosky, 2018)

Diagnósticos Diferenciales

- Hematoma subdural agudo en el servicio de urgencias

- Epilepsia y convulsiones

- Genética de la hiperamonemia

- Lesión craneal

- Manifestaciones neurológicas de la demencia vascular.

- Encefalomielitis paraneoplásica

- Amnesia Global Transitoria

- Neuropatía urémica

- Encefalitis viral

- Meningitis viral. (Korabathina, Benbadis, \& Likosky, 2018)

Complicaciones 


\section{Manejo en intoxicación por metanol}

Vol. 3, núm. 3., (2019)

Jenny Irene Plasencia Criollo; Fátima Victoria Gualotuña Cueva; María Jhaneth Delgado Angamarca; Sixto Duberli Paucar Llapapasca

De acuerdo al aporte hecho en la obra antes referida, ha de comprenderse que aparte de los efectos un tanto más inmediatos que se han mencionado, es posible que este tipo de intoxicaciones produzcan otro tipo de daños más profundos, entre los que figuran:

\section{Pérdida de la visión}

El mecanismo por el cual el metanol causa toxicidad en el sistema visual no se conoce bien. El ácido fórmico, el metabolito tóxico del metanol, es responsable de la toxicidad ocular en modelos animales y se presume que es responsable en estudios en humanos.

Los niveles séricos de metanol superiores a $20 \mathrm{mg} / \mathrm{dL}$ se correlacionan con la lesión ocular. Los cambios en el fondo de ojo son notables solo unas pocas horas después de la ingestión de metanol y van desde edema retiniano en la región perimacular hasta el fondo completo. El edema de disco óptico y la hiperemia se observan dentro de las 48 horas.

La lesión visual puede prevenirse con una terapia antídoto inmediata o mediante la eliminación de los metabolitos del sistema con hemodiálisis; Sin embargo, este no es siempre el caso.

\section{Trastornos del movimiento}

El deterioro motor parkinsoniano se ha descrito en algunos sobrevivientes a largo plazo de intoxicación por metanol. Se cree que esto se debe a la predilección del ácido fórmico por acumularse en altas concentraciones dentro del putamen, pero las razones de este fenómeno no 


\section{Manejo en intoxicación por metanol}

Vol. 3, núm. 3., (2019)

Jenny Irene Plasencia Criollo; Fátima Victoria Gualotuña Cueva; María Jhaneth Delgado

Angamarca; Sixto Duberli Paucar Llapapasca

están claras. Una razón propuesta es que el ácido fórmico tiene la capacidad de dañar las vías dopaminérgicas y aumentar la actividad enzimática de la dopa-B-hidroxilasa.

El inicio de los síntomas generalmente se retrasa varias semanas después de la exposición al metanol. Se han descrito bien los síntomas parkinsonianos comunes, como temblor, rigidez de la rueda dentada, postura inclinada, marcha arrastrada e hipocinesia. Además, se ha establecido el desarrollo de signos de distonía y tracto corticoespinal.

Varios informes de casos han indicado una respuesta sintomática a los agentes antiparkinsonianos estándar, particularmente levodopa, amantadina y bromocriptina.

También se han reportado espasmos musculares en la intoxicación por metanol. Como se esperaba, este síntoma responde mal a la terapia tradicional.

En raras ocasiones, se han observado lesiones en las regiones lobulares del cerebro y el cerebelo. (Korabathina, Benbadis, \& Likosky, 2018)

\section{Tratamiento}

1. Descontaminación: no se recomienda hacer lavado gástrico ni administrar carbón activado. 


\section{Manejo en intoxicación por metanol}

Vol. 3, núm. 3., (2019)

Jenny Irene Plasencia Criollo; Fátima Victoria Gualotuña Cueva; María Jhaneth Delgado Angamarca; Sixto Duberli Paucar Llapapasca

2. Antídotos: inhiben de forma competitiva la alcohol deshidrogenasa y por consiguiente el metabolismo del metanol.

2.1. Etanol: dosis de carga en personas conscientes (y sin riesgo de hemorragia digestiva $\rightarrow$ VO 2,5 ml de solución al $40 \% / \mathrm{kg}$; en inconscientes iv. solución de etanol al $10 \%$ en glucosa al $5 \% 10 \mathrm{ml} / \mathrm{kg}$ en $30 \mathrm{~min}$. Dosis de mantenimiento 1,5 ml/kg/h de la solución de etanol al $10 \%$ iv., en personas adictas 2-3 ml/kg/h, durante la hemodiálisis 3-4 ml/kg/h. El objetivo de la terapia es una concentración de etanol en sangre 100-150 mg/dl (1-1,5 \%o).

2.2. Fomepizol: se administra iv., en infusión lenta (durante $30 \mathrm{~min}$ ) en $250 \mathrm{ml}$ de $\mathrm{NaCl}$ al 0,9 \% o solución de glucosa al $5 \%$. Dosis de carga $15 \mathrm{mg} / \mathrm{kg}$. Dosis de mantenimiento inicial $10 \mathrm{mg} / \mathrm{kg}$, con un total de 4 dosis cada $12 \mathrm{~h}$, seguido de $15 \mathrm{mg} / \mathrm{kg}$ cada $12 \mathrm{~h}$. En la diálisis $10-15 \mathrm{mg} / \mathrm{kg}$, cada $4 \mathrm{~h}$.

2.3. Ácido folínico (folinato cálcico) 50-70 mg iv. o ácido fólico $50 \mathrm{mg}$ VO (o a través de una sonda nasogástrica en personas inconscientes) cada 4-6h, para aumentar la eliminación del ácido fórmico.

3. Medidas para acelerar la eliminación: hemodiálisis. Indicaciones: concentración de metanol en sangre $>50 \mathrm{mg} / \mathrm{dl}$, concentración de metanol en sangre $<50 \mathrm{mg} / \mathrm{dl}$ con acidosis metabólica concomitante, alteraciones de la visión, acidosis metabólica grave, condición clínica severa y falta de mejoría a pesar del tratamiento, desequilibrio electrolítico grave en el curso de la intoxicación. Continuar la hemodiálisis hasta que todo el metanol haya sido eliminado del organismo y haya desaparecido la acidosis (hasta más de 10 h).

Revista Científica Mundo de la Investigación y el Conocimiento. 3 (3). pp. 842-869 


\section{Manejo en intoxicación por metanol}

Vol. 3, núm. 3., (2019)

Jenny Irene Plasencia Criollo; Fátima Victoria Gualotuña Cueva; María Jhaneth Delgado

Angamarca; Sixto Duberli Paucar Llapapasca

4. Tratamiento sintomático: mantener las funciones básicas del organismo y corregir los trastornos existentes. Tratamiento inicial de la acidosis metabólica: al principio 1-2 mmol/kg de $\mathrm{NaHCO} 3$ iv. (en la primera etapa de intoxicación $\geq 400-600 \mathrm{mmol}$ ) en todos los pacientes con $\mathrm{pH}$ arterial $<7,3$ con el objetivo del lograr un $\mathrm{pH}>7,35$. En la mayoría de los casos, a pesar de la administración de NaHCO3 es necesario realizar la hemodiálisis. (Empendium, 2019)

Cabría destacar que las novedosas técnicas de imagen actualmente facilitan una comprensión mejorada de las manifestaciones clínicas de la intoxicación por metanol. De esta manera se han hecho cada vez más habituales el diagnóstico de las complicaciones neurológicas, no obstante, esto también se debe al oportuno reconocimiento de la toxicidad y a las mejoras en la atención de apoyo.

La atención médica inmediata es clave para evitar complicaciones secundarias a la intoxicación por metanol. La terapia de apoyo tiene como objetivo iniciar el manejo de las vías respiratorias, corregir las alteraciones electrolíticas y proporcionar una hidratación adecuada.

La acidosis metabólica en el envenenamiento por metanol puede requerir la administración de bicarbonato y ventilación asistida. El bicarbonato potencialmente puede revertir los déficits visuales. Además, el bicarbonato puede ayudar a disminuir la cantidad de ácido fórmico activo.

La terapia antídoto, a menudo usando etanol o fomepizol, está dirigida a retrasar el metabolismo del metanol hasta que el metanol se elimine del sistema del paciente, ya sea de forma natural o mediante diálisis. Al igual que el metanol, el etanol se metaboliza por ADH, pero 


\section{Manejo en intoxicación por metanol}

Vol. 3, núm. 3., (2019)

Jenny Irene Plasencia Criollo; Fátima Victoria Gualotuña Cueva; María Jhaneth Delgado Angamarca; Sixto Duberli Paucar Llapapasca

la afinidad de la enzima por el etanol es 10-20 veces mayor que por el metanol. Fomepizol también se metaboliza por ADH; sin embargo, su uso es limitado debido al alto costo y la falta de disponibilidad.

La hemodiálisis puede eliminar fácilmente el metanol y el ácido fórmico. Las indicaciones para este procedimiento incluyen: a) más de $30 \mathrm{ml}$ de metanol ingerido, b) nivel de metanol en suero superior a $20 \mathrm{mg} / \mathrm{dL}$, c) observación de complicaciones visuales y d) ninguna mejora en la acidosis a pesar de las infusiones repetidas de bicarbonato de sodio. (Korabathina, Benbadis, \& Likosky, 2018)

\section{Manejo Clínico}

Casos sospechosos.

Valoración de paciente.

Ingreso a Triage:

- Evaluar estado de Consciencia Escala de Glasgow (Ver escala)

- Tomar Signos Vitales

- Historia Clínica (1. Fecha de última ingesta. 2. Estuvo bebiendo con alguien que esta intoxicado)

Si paciente no verbaliza, conversar con familiar.

Medidas generales. 


\section{Manejo en intoxicación por metanol}

Vol. 3, núm. 3., (2019)

Jenny Irene Plasencia Criollo; Fátima Victoria Gualotuña Cueva; María Jhaneth Delgado

Angamarca; Sixto Duberli Paucar Llapapasca

Ingresar a atención rápida y evaluar cada 2 horas

En caso de no existir tolerancia oral colocar vía periférica, catéter 18.

Hidratación con Solución Salina al 0.9\% para mantener diuresis mayores de $0.5 \mathrm{ml} / \mathrm{Kg} / \mathrm{hora}$

\section{Solicitar GSA}

Si no existen alteración clínica ni en la GSA en 4 a 6 horas dar el alta, realizar seguimiento telefónico.

Evaluación de signos y síntomas de alerta.

- Taquipnea

- Alteración del estado de conciencia.

- Reacción a la luz fotofobia, midriasis, visión borrosa y otros trastornos visuales agudos progresivos.

- Hipoglucemia (Glucosa capilar)

- Si paciente presenta uno de los síntomas de alerta, manejar como caso probable y en una situación de deterioro de estado de conciencia o

- Escala de Glasgow < 10, taquipnea, cianosis, shock; ingresar inmediatamente a UCI o área critica.

Manejo de casos probables. 


\section{Manejo en intoxicación por metanol}

Vol. 3, núm. 3., (2019)

Jenny Irene Plasencia Criollo; Fátima Victoria Gualotuña Cueva; María Jhaneth Delgado Angamarca; Sixto Duberli Paucar Llapapasca

- Ingreso a observación de clínica.

- $\mathrm{ABCD}$.

- Monitorización continua.

- Colocar vía periférica, catéter 18.

- Hidratación con Solución Salina al $0.9 \%$ para mantener diuresis mayores de $0.5 \mathrm{ml} / \mathrm{Kg} / \mathrm{hora}$.

- Control de ingesta y excreta.

- Solicitud de: GSA, ácido láctico, hemograma completo, nitrógeno ureico, creatinina, sodio, cloro, potasio, glucosa, amilasa, perfil hepático, CPK, orina completa, niveles de metanol y etanol en sangre.

- Rx de tórax, si se sospecha de neumonía por aspiración.

- $\quad$ ECG de 12 derivaciones.

- Valoración por oftalmología.

- $\quad$ TAC simple de encéfalo si el Glasgow $<12$

Para ayudar al diagnóstico se utiliza el cálculo del anión gap y del osmolar gap, encontrándose el anión gap elevado, se puede estimar de forma aproximada la cantidad de metanol en mg/dl calculando el osmol gap y multiplicando este por un factor de corrección de 3,2 .

- Anión gap: $[\mathrm{Na}]+[\mathrm{K}]-([\mathrm{Cl}]+[\mathrm{HCO} 3])$

- Valor normal: 8- $16 \mathrm{mEq} / \mathrm{L}$. 


\section{Manejo en intoxicación por metanol}

Vol. 3, núm. 3., (2019)

Jenny Irene Plasencia Criollo; Fátima Victoria Gualotuña Cueva; María Jhaneth Delgado

Angamarca; Sixto Duberli Paucar Llapapasca

- La osmolaridad calculada es: OC: $2[\mathrm{Na}]+\mathrm{BUN} / 2.8+$ glucosa/18 o $2[\mathrm{Na}]+$ urea/5.6 + glucosa/18

Valor normal: 10-15 mOsm/L.

Tener en cuenta que en el curso temporal de la intoxicación por metanol el osmol gap va descendiendo y el anión gap aumentando.

En ausencia de cuantificación de niveles de metanol, anión y osmol gap normales y ausencia de sintomatología, se procede con el descarte de una ingesta de alcohol metílico.

La gravedad de la intoxicación se clasifica de acuerdo con los niveles de metanol en la sangre, que se correlacionan con la clínica en las siguientes formas:

Tabla 1. Niveles de intoxicación por metanol

\begin{tabular}{|c|c|c|}
\hline Leve & Moderada & Grave \\
\hline \multirow{6}{*}{$\begin{array}{l}\text { Metanolemia (metanol en } \\
\text { sangre) menor de } 0.1 \\
\mathrm{~g} / \mathrm{L} \text {. Sensación de fatiga, } \\
\text { náuseas, epigastralgias, } \\
\text { cefalea y transtornos } \\
\text { visuales de percepción o } \\
\text { acomodación. }\end{array}$} & Metanolemia entre $0.1-0.5$ & \multirow{6}{*}{$\begin{array}{l}\text { Metanolemia mayor de } \\
0.5 \mathrm{~g} / \mathrm{L} \text { a } 1 \mathrm{~g} / \mathrm{L} \text {. Paro } \\
\text { comatoso, respiración } \\
\text { rápida y superficial, } \\
\text { convulsiones, cianosis } \\
\text { periférica y central, } \\
\text { hipotensión, edema de } \\
\text { papila. }\end{array}$} \\
\hline & g/L. Vómitos, & \\
\hline & expresiones de embriaguez, & \\
\hline & especialmente si la & \\
\hline & & \\
\hline & $\begin{array}{l}\text { intoxicación es mixta } \\
\text { (etanol-metanol), piel fría y }\end{array}$ & \\
\hline
\end{tabular}




\section{Manejo en intoxicación por metanol}

Vol. 3, núm. 3., (2019)

Jenny Irene Plasencia Criollo; Fátima Victoria Gualotuña Cueva; María Jhaneth Delgado Angamarca; Sixto Duberli Paucar Llapapasca

\begin{tabular}{|c|c|}
\hline $\begin{array}{l}\text { sudorosa, visión borrosa y se } \\
\text { presenta taquipnea, } \\
\text { tratando de hacer } \\
\text { compensación respiratoria } \\
\text { de } \\
\text { la acidosis metabólica. }\end{array}$ & $\begin{array}{l}\text { Las metanolemias } \\
\text { superiores a } 1 \mathrm{~g} / \mathrm{L} \text { se } \\
\text { consideran letales. }\end{array}$ \\
\hline
\end{tabular}

Nota: Adaptada de Ministerio de Salud Pública Ecuador (2017)

Ashurst \& Nappe (2019) Las opciones de tratamiento para la toxicidad del metanol incluyen cuidados de apoyo, fomepizol (Antizole, 4-Methylpyrazole o 4MP), etanol, diálisis y, en teoría, folato. El fomepizol es el antídoto para los alcoholes tóxicos, y su mecanismo de acción es la inhibición de la alcohol deshidrogenasa. El etanol también se puede utilizar terapéuticamente para inhibir la alcohol deshidrogenasa cuando el fomepizol no está disponible. Hay ventajas y desventajas de cualquiera de los tratamientos. Fomepizol se dosifica más fácilmente, no causa ninguna embriaguez, inhibe fuertemente la alcohol deshidrogenasa, pero es bastante costoso. El etanol es menos costoso, pero es más difícil de dosificar con precisión, requiere un estrecho control de la concentración de etanol en suero y provoca embriaguez que puede requerir un control intensivo.

Las indicaciones para el tratamiento incluyen una concentración elevada de metanol y acidosis severa o progresiva, a pesar de la reanimación, con sospecha clínica de ingestión de metanol. Las recomendaciones con respecto a las concentraciones específicas de metanol varían con respecto a cuándo comenzar el tratamiento. Las recomendaciones más conservadoras son comenzar el tratamiento si la concentración de metanol es mayor de 20 a $25 \mathrm{mg} / \mathrm{dL}$. Sin embargo, si la acidosis metabólica es leve o no está presente, y no hay evidencia de toxicidad en los órganos terminales, entonces una concentración de metanol de $32 \mathrm{mg} / \mathrm{dL}$ es un punto de 


\section{Manejo en intoxicación por metanol}

Vol. 3, núm. 3., (2019)

Jenny Irene Plasencia Criollo; Fátima Victoria Gualotuña Cueva; María Jhaneth Delgado

Angamarca; Sixto Duberli Paucar Llapapasca

partida apropiado para el tratamiento, ya que los cálculos molares indican que esto se correlacionaría con un máximo de $10 \mathrm{mmol}$. / L de un metabolito tóxico (formiato). Esto no debería representar solo un déficit de base de más de $10 \mathrm{mmol} / \mathrm{L}$ o una cantidad tóxica de metabolito. (Nota: el límite de tratamiento basado en molar para etilenglicol es $62 \mathrm{mg} / \mathrm{dL}$; ver capítulo etilenglicol). Cuando no se puede alcanzar la concentración de metanol, una indicación apropiada para el tratamiento debe ser cuando el bicarbonato progresa por debajo de $15 \mathrm{mmol} / \mathrm{L}$ o si hay evidencia de toxicidad retiniana. Después del tratamiento empírico con fomepizol, hay 12 horas en las que se detiene el metabolismo del metanol, lo que permite un tiempo adecuado para obtener una concentración de metanol y organizar la diálisis, si es necesario.

Fomepizol o etanol sirven como inhibidores de la alcohol deshidrogenasa para detener la conversión de metanol a su metabolito tóxico, el formiato. Cuando se inhibe la alcohol deshidrogenasa, el aclaramiento de metanol se prolonga de aproximadamente $8,5 \mathrm{mg} / \mathrm{dL} / \mathrm{h}$ a una vida media efectiva de 45 a 90 horas. Fomepizol se administra por vía intravenosa, con una dosis de carga de $15 \mathrm{mg} / \mathrm{kg}$, y luego dosis de mantenimiento de $10 \mathrm{mg} / \mathrm{kg}$ cada 12 horas durante 4 dosis o hasta que la concentración de metanol sea inferior a $32 \mathrm{mg} / \mathrm{dL}$ con un estado ácido-base normal. Si se requiere una dosificación adicional más allá de 4 dosis de mantenimiento, la dosificación aumenta a $15 \mathrm{mg}$ / $\mathrm{kg}$ cada 12 horas debido a la autoinducción de un aumento del metabolismo. Durante la diálisis, el fomepizol debe dosificarse cada 4 horas, ya que es dializable.

La dosificación de etanol es más complicada, difícil de controlar y tiene el efecto secundario adicional de la embriaguez. El etanol puede administrarse por vía intravenosa u oral. Sin embargo, solo se debe administrar si el fomepizol no está disponible, ya que sería 


\section{Manejo en intoxicación por metanol}

Vol. 3, núm. 3., (2019)

Jenny Irene Plasencia Criollo; Fátima Victoria Gualotuña Cueva; María Jhaneth Delgado Angamarca; Sixto Duberli Paucar Llapapasca

inapropiado causar que el paciente se embriague durante un período tan prolongado. Cuando se trata con etanol, la concentración sérica terapéutica objetivo es un rango de 80 a 120 mg / dL. El formulario de etanol intravenoso suele ser del 10\%, y se calcula una dosis de carga utilizando el producto de la concentración plasmática objetivo $(C=100 \mathrm{mg} /$ dL), el volumen de distribución de etanol $(\mathrm{V}=0.6 \mathrm{~L} / \mathrm{kg})$ y el peso del paciente. La dosificación de mantenimiento se basa entonces en la tasa de eliminación. Empíricamente, se puede administrar etanol intravenoso al $10 \%$ con una dosis de carga de $8 \mathrm{ml} / \mathrm{kg}$ durante 30 a 60 minutos, seguido de una dosis de mantenimiento de 1 a $2 \mathrm{ml} / \mathrm{kg}$ por hora. La dosis de mantenimiento se duplica durante la diálisis. La dosificación oral se puede calcular usando la ecuación anterior para las concentraciones de alcohol en suero usando $100 \mathrm{mg} / \mathrm{dL}$ para la concentración en suero y luego resolviendo la cantidad ingerida. Empíricamente, se puede administrar etanol oral al 50\% (100 pruebas) con una dosis de carga de $2 \mathrm{ml} / \mathrm{kg}$ seguido de 0.2 a $0.4 \mathrm{ml} / \mathrm{kg}$ por hora. La dosis de mantenimiento se duplica durante la diálisis.

Los pacientes con una ingestión de metanol tóxico deben ser considerados para la hemodiálisis. Debido a su bajo volumen de distribución y la falta de unión a proteínas, tanto el metanol como el metabolito tóxico, el formiato, son dializables. La hemodiálisis a menudo es beneficiosa para la toxicidad del metanol porque puede disminuir significativamente la duración de la estadía del paciente. Una vez que se inhibe la alcohol deshidrogenasa, el aclaramiento de metanol se prolonga de aproximadamente $8,5 \mathrm{mg} / \mathrm{dL}$ por hora a una vida media efectiva de 45 a 90 horas. Sin embargo, la única indicación absoluta de hemodiálisis en la toxicidad del metanol es una nueva discapacidad visual en presencia de acidosis metabólica. Las indicaciones relativas para la hemodiálisis incluyen una concentración de metanol superior a $50 \mathrm{mg} / \mathrm{dL}$, acidosis 


\section{Manejo en intoxicación por metanol}

Vol. 3, núm. 3., (2019)

Jenny Irene Plasencia Criollo; Fátima Victoria Gualotuña Cueva; María Jhaneth Delgado

Angamarca; Sixto Duberli Paucar Llapapasca

metabólica grave resistente a la reanimación, antecedentes de ingestión de una dosis letal de $1 \mathrm{~g} /$ $\mathrm{kg}$, insuficiencia renal,

El tratamiento adicional de la toxicidad por metanol incluye ácido fólico. La administración de folato es de beneficio teórico, ya que puede mejorar el metabolismo del metabolito tóxico, formiato, a dióxido de carbono y agua.

La admisión a la unidad de cuidados intensivos es apropiada cuando un paciente tiene una sintomatología severa, trastornos metabólicos severos, necesita diálisis o está siendo tratado con etanol como antídoto.

\section{Conclusión.}

La intoxicación por metanol, aunque aún puede considerarse como relativamente rara no deja de ser particularmente trascendente. Así se ha constatado, generalmente, en la literatura consultada, pues en múltiples estudios se menciona que, del total de consultas en emergencias, sólo se llega a medir menos del $1 \%$ de estos casos.

Clínicamente, se caracteriza por manifestarse con distintos síntomas, que, dependiendo de cada caso, pudiera referirse fatiga, náuseas, vómitos, dolor abdominal, hipotensión, problemas sensoriales (inclusive, taquicardia, taquipnea, en base a ello), problemas de visión (en el examen oftalmológico pudiera dejarse ver una papilitis óptica, que según estudios se da en el $10 \%$ de los casos), midriasis y nistagmo, entre otros. El ácido fórmico, como principal metabolito del metanol, viene siendo el principal responsable de la mayoría de las anormalidades clínicas de esta enfermedad. 


\section{Manejo en intoxicación por metanol}

Vol. 3, núm. 3., (2019)

Jenny Irene Plasencia Criollo; Fátima Victoria Gualotuña Cueva; María Jhaneth Delgado Angamarca; Sixto Duberli Paucar Llapapasca

Del análisis e interpretación de recientes y variados estudios, se logró comprender que los autores mantienen la idea de manejar esta condición con terapias combinadas, preferiblemente, en las que se deben incluir: la administración de un inhibidor, como es el caso del fomepizol, y a falta de este bien pudiera indicarse el etanol por su efectividad. Otros especialistas coinciden en restringir la hemodiálisis a circunstancias específicas y severas. En resumen, la combinación de tratamientos favorecerá significativamente la delimitación en cuanto a la exposición medicamentosa y la reducción de los días de hospitalización; y en definitiva, basándose en la naturaleza inespecífica de muchos de los síntomas y signos de esta condición, se hace necesario alcanzar un alto grado de sospecha antes de iniciar el correspondiente tratamiento, no obstante, la atención médica inmediata es clave para evitar complicaciones secundarias, y de allí pues, el tratamiento óptimo requiere un reconocimiento anticipado y un rápido inicio de una acertada terapia.

\section{Bibliografía.}

Ashurst, J., \& Nappe, T. (15 de Marzo de 2019). StatPearls [Internet] (Electrónica ed.). Treasure Island, Florida, USA: StatPearls Publishing LLC. Recuperado el 15 de 09 de 2019, de www.ncbi.nlm.nih.gov/books/NBK482121/

Borke, J. (12 de Enero de 2019). Medlineplus. Recuperado el 15 de 09 de 2019, de medlineplus.gov/spanish: https://medlineplus.gov/spanish/ency/article/002680.htm

Contreras, C., Lira, H., Contreras, K., \& Gala, D. (Abril de 2019). Magnitud y características de la intoxicación por alcohol metílico. Hospital Nacional Dos de Mayo. Horizonte Médico, 19(1), 59-66. doi:doi.org/10.24265/horizmed.2019.v19n1.10

Copaja, M. (09 de 09 de 2018). Metanol: Toxicidad, regulación y análisis. Recuperado el 14 de 09 de 2019, de https://agqlabs.cl/wp-content/uploads/Metanol-toxicidad-regulacion-yanalisis.pdf

Empendium. (01 de 10 de 2019). Alcohol metílico (metanol). Recuperado el 05 de 09 de 2019, de https://empendium.com/manualmibe/chapter/B34.II.20.2.2. 


\section{Manejo en intoxicación por metanol}

Vol. 3, núm. 3., (2019)

Jenny Irene Plasencia Criollo; Fátima Victoria Gualotuña Cueva; María Jhaneth Delgado

Angamarca; Sixto Duberli Paucar Llapapasca

GreenFacts. (13 de 09 de 2019). Metanol. Recuperado el 14 de 09 de 2019, de https://www.greenfacts.org/es/glosario/mno/metanol.htm

Korabathina, K., Benbadis, S., \& Likosky, D. (02 de 08 de 2018). Methanol Toxicity. Recuperado el 14 de 09 de 2019, de https://emedicine.medscape.com/article/1174890overview

Ministerio de Salud Pública Ecuador. (2017). Lineamientos Operativos para el Manejo de Pacientes con Intoxicación por Metanol. Lineamientos Operativos para el Manejo de Pacientes con Intoxicación por Metanol en los establecimientos del Ministerio de Salud Pública, Ministerio de Salud Pública Ecuador, 22 pp. (D. N. Hospitales, Ed.) Quito, Ecuador: Subsecretaría Nacional de Provisión de Servicios de Salud. Recuperado el 15 de 09 de 2019, de http://hospitalgeneralchone.gob.ec/wp-content/uploads/2018/09/snpsslineamientos_manejo_de_intoxicaci\%D0\%B2n_por_metanol._msp-2017.pdf

NIOSH. (24 de Junio de 2019). Centros de Control y Prevención de Enfermedades. Recuperado el 15 de 09 de 2019, de https://www.cdc.gov/niosh/topics/methyl-alcohol/

Orphanet. (02 de 08 de 2019). Intoxicación por metanol. Recuperado el 14 de 09 de 2019, de https://www.orpha.net/consor/cgi-bin/OC_Exp.php?Expert=31825\&lng=ES 